\title{
Quatre disques compacts et un microsillon de musique villageoise roumaine
}

Jacques Bouët

\section{OpenEdition}

Journals

Édition électronique

URL : http://journals.openedition.org/ethnomusicologie/2366

ISSN : 2235-7688

Éditeur

ADEM - Ateliers d'ethnomusicologie

Édition imprimée

Date de publication : 1 janvier 1989

Pagination : 297-303

ISBN : 2-8257-0178-5

ISSN : 1662-372X

\section{Référence électronique}

Jacques Bouët, «Quatre disques compacts et un microsillon de musique villageoise roumaine »,

Cahiers d'ethnomusicologie [En ligne], 2 | 1989, mis en ligne le 15 septembre 2011, consulté le 26 avril 2019. URL : http://journals.openedition.org/ethnomusicologie/2366

Ce document a été généré automatiquement le 26 avril 2019

Article L.111-1 du Code de la propriété intellectuelle. 


\section{Quatre disques compacts et un microsillon de musique villageoise roumaine}

Jacques Bouët

\section{RÉFÉRENCE}

Roumanie: Musique de villages (Olténie - Moldavie - Transylvanie). Coffret de trois disques compacts (ADD) accompagné d'une notice explicative rédigée par Speranza Rădulescu et illustrée de magnifiques photographies anciennes. Références : VDE, CD 537/539, Disques VDE Gallo, CH-1407 Donneloye. Édition établie en 1988 par repiquage d'un matériel musical tiré des archives de l'ICED de Bucarest. Les enregistrements ont été réalisés de 1933 à 1943 par Constantin Brăiloiu et ses collaborateurs (notamment Tiberiu Alexandru pour les enregistrements en studio). Sélection musicale : Speranza Rădulescu (ICED) et Laurent Aubert (AIMP).

Roumanie: Musique des Tsiganes de Valachie - Le taraf de Clejani. Un disque compact ADD publié sous le triple label Radio Télévision de la Suisse romande (RTSR), Archives internationales de musique populaire (AIMP, Genève) et OCORA Radio France. Distributeur Harmonia Mundi, Paris, février 1988. Enregistrement réalisé le 8 décembre 1986 dans le studio de l'Institut de recherches ethnologiques et dialectologiques (ICED) à Bucarest. Réalisation : Laurent Aubert, Thierry Fischer. Texte de présentation : Laurent Aubert. Conseiller scientifique : Speranza Rădulescu. Prise de son : Thierry Fischer. Transylvanian Harmonies. Performers in the Past. Un microsillon ( 33 tours, $30 \mathrm{~cm}$ ) d'enregistrements effectués en 1941 par Constantin Brăiloiu et Tiberiu Alexandru à Bucarest. Publication de l'Institut de recherches ethnologiques et dialectologiques (ICED) de Bucarest, sous la responsabilité éditoriale de Tiberiu Alexandru. Notice rédigée par Tiberiu Alexandru. The National Collection of Folklore, Disque ELECTRECORD, EPE 003319, Bucarest, 1984. 
1 Il était à craindre que la musique villageoise roumaine qui avait connu une renommée justifiée dans les années soixante-dix et quatre-vingt, soit à plus ou moins brève échéance reléguée aux oubliettes si elle n'entrait pas très vite sur le marché du disque compact. Voilà ce risque écarté : les Archives internationales de musique populaire (AIMP) du Musée d'ethnographie de la Ville de Genève, qui furent fondées par l'ethnomusicologue roumain Constantin Brăiloiu, viennent de rendre un hommage direct à leur fondateur en consacrant un coffret de trois disques compacts à des repiquages d'enregistrements effectués par Brăiloiu lui-même dans les années trente et quarante en Roumnaie. Le responsable actuel des AIMP, Laurent Aubert, qui est à l'origine de cette parution, vient également de publier un disque compact consacré au répertoire d'un ensemble tsigane du village de Clejani en Valachie.

2 Par ailleurs, l'Institut de recherches ethnologiques et dialectologiques (ICED) de Bucarest a récemment édité un microsillon composé d'enregistrements - effectués en 1941 par Constantin Brăiloiu et Tiberiu Alexandru à Bucarest - de musiques de danse transylvaines pour petits ensembles à cordes. L'éditeur en est Tiberiu Alexandru.

Roumanie : Musique de villages (Olténie - Moldavie - Transylvanie). Coffret de trois disques compacts (ADD) accompagné d'une notice explicative rédigée par Speranza Rădulescu et illustrée de magnifiques photographies anciennes. Références : VDE, CD 537/539, Disques VDE Gallo, CH-1407 Donneloye. Édition établie en 1988 par repiquage d'un matériel musical tiré des archives de l'ICED de Bucarest. Les enregistrements ont été réalisés de 1933 à 1943 par Constantin Brǎiloiu et ses collaborateurs (notamment Tiberiu Alexandru pour les enregistrements en studio). Sélection musicale : Speranza Rădulescu (ICED) et Laurent Aubert (AIMP).

3 Fruit d'une heureuse collaboration entre une Roumaine et un Suisse, tous deux ethnomusicologues, ce coffret comprend trois disques dont chacun est consacré à deux aires géoculturelles définies (village ou groupe de villages) bien localisées dans une des trois grandes provinces de Roumanie. Ainsi a pu être évité le piège de l'échantillonnagesurvol des anthologies "ancienne manière" qui, le plus souvent, laissaient leurs auditeurs sur de pénibles frustrations.

4 A titre d'exemple, le premier disque, entièrement consacré au département de Gorj en Olténie, est subdivisé en deux parties, la première comprenant douze pièces provenant de sept villages différents, la seconde, dix enregistrées dans le seul village de Runc. Les pièces choisies sont tout à fait représentatives du répertoire: plusieurs versions de " chant long », instrumentales et vocales, diverses danses instrumentales et mélodies de danse, deux chants funèbres, un chant de noce, un récit épique chanté (ballade), la danse des căluşari (rite de guérison courant en Olténie).

5 L'auditeur a donc à sa disposition une documentation sonore quasi monographique que le texte de la notice permet de replacer dans la situation d'enregistrement et dans le contexte social. En outre, on peut suivre le sens des textes chantés, car pour chacun d'eux (à une exception près), les éditeurs ont donné une version originale en roumain, assortie d'une traduction française et anglaise.

6 Un souci de représentativité géoculturelle a donc présidé à la sélection des enregistrements, et c'est là l'un des principaux mérites de cette publication. 
Elle en a de nombreux autres. Ainsi, pour ce qui est du montage, on perçoit, en ce qui concerne le disque consacré à Gorj (disque A), une intention sous-jacente pleine de conséquences. En effet, plusieurs versions du "chant long", que Brăiloiu appelait luimême "à l'olténienne ", sont placées à la suite l'une de l'autre: les premières (plages 1 et 2), instrumentales (cornemuse et flûte à bourdon gutturale "à la manière des bergers »), sont suivies de versions vocales (solo sans accompagnement, plages $3,4,5,6$ ). Puis vient une version «à la manière des lăutari »: un solo vocal féminin - précédé d'un prélude instrumental (version violonistique ornementée d'un chant long) - est superposé à un accompagnement harmonique exécuté par un taraf (guitare, violon, contrebasse). Un ensemble de données essentielles est ainsi mis à la disposition de l'auditeur et lui permet de saisir le processus de stratification du répertoire villageois roumain et celui de structuration de la musique des lăutari. C'est là d'ailleurs une problématique à laquelle Speranta Rădulescu a consacré une partie de son bel ouvrage sur le taraf ${ }^{1}$, et sur lequel elle revient ici, montage sonore à l'appui, de façon que chacun puisse s'en faire une idée par lui-même. On constate ainsi à quel point les ornements vocaux (hăiulit), si caractéristiques de Gorj comme de toute l'olténie, sont proches de ceux qu'exécutent les instrumentistes à la cornemuse ou à la flûte. Qui est l'imitateur de l'autre? L'instrumentiste, ou le chanteur? Sans doute y a-t-il eu va-et-vient de l'un à l'autre.

Tsărăneasca, danse de Bucovine.

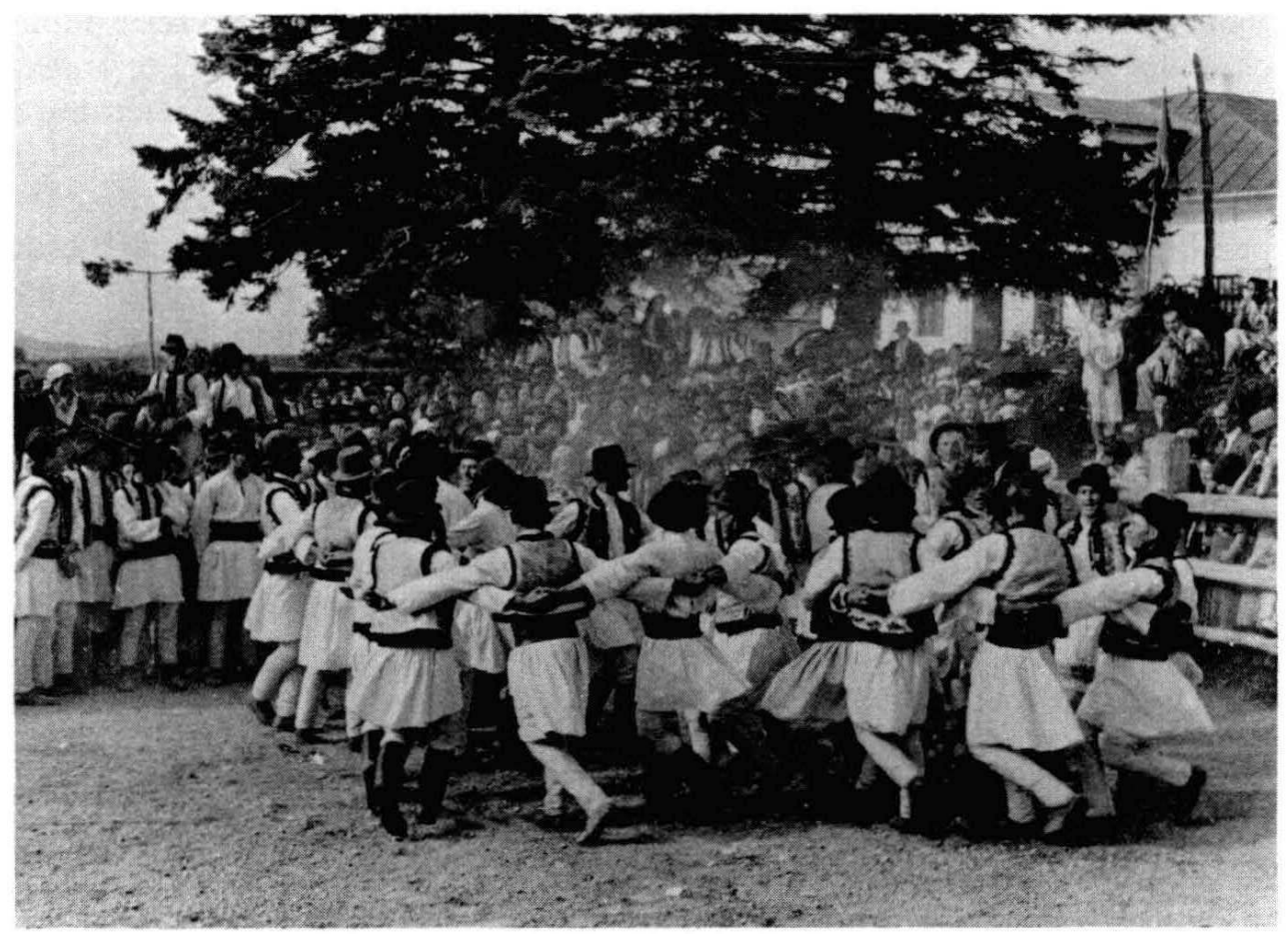

Photo : Archives ICED, Bucarest.

Quant à l'agencement polyphonique de l'accompagnement poly-instrumental (plage 7), il est manifestement d'une conception toute proche de celui de la cornemuse qu'on entend plage 1, car la basse du taraf, tout comme le bourdon oscillant de la même cornemuse, alterne systématiquement du degré fondamental à la quarte inférieure tout au long de la pièce en question. On tient là un indice de nature à accréditer l'hypothèse selon laquelle les éléments paysans auraient joué, dans la structuration du répertoire, un rôle plus 
déterminant que les éléments turcs et moyen-orientaux que, de toute façon, les Tsiganes n'ont pu manquer de véhiculer dans les Balkans pendant plus de cinq siècles de domination ottomane. Cela d'autant plus que le «chant long à l'olténienne » qui a servi de mélodie à nombre de récits chantés, n'est pas moins courant dans le répertoire citadin que dans celui des villages. Cependant, là encore, on se fourvoierait si l'on cherchait à expliquer l'état actuel des musiques de lăutari par un mouvement à sens unique des villages aux faubourgs ou des faubourgs au villages, car selon toute vraisemblance, il est le résultat de multiples emprunts réciproques et d'une synthèse complexe.

On trouvera à la plage 20 du disque A une ballade magnifiquement chantée, préludée et accompagnée. Peut-on rencontrer encore aujourd'hui de tels chanteurs? Un enregistrement tout à fait semblable à celui-ci a été effectué en Valachie par Bernard Lortat-Jacob et moi-même en 1981, qui n'a pu être publié pour des raisons purement techniques. Cela veut dire que cinquante années après les enquêtes de Brăiloiu, la tradition qu'on croyait déjà mourante à l'époque s'est conservée. Cela va-t-il durer? En tout cas, on ne peut que se réjouir de voir gravée une pareille merveille sur un support réputé inusable.

De fort belles plages sont consacrées à un genre qu'on assimile parfois au «chant long " bien qu'il s'en écarte sensiblement : la doina de Bucovine (Moldavie du nord), disque B. Les textes de ces doine, qu'on a intérêt à suivre en écoutant la musique, véhiculent une mélancolie spécifique à laquelle la mélodie donne une qualité toute particulière. On ne peut s'empêcher de songer au Voyage d'hiver de Müller et Schubert pour ce qui est des moyens expressifs mis en œuvre. On est donc de ce point de vue en présence d'un genre qui, bien qu'exclusivement oral et paysan, relève peut-être plus du lied romantique que des musiques vocales dont l'ethnomusicologie se préoccupe en priorité, mais il eût été dommage pour une telle raison de l'écarter de la sélection. On ne peut donc que se réjouir, une fois de plus, que les co-éditeurs aient jugé bon de lui consacrer une bonne dizaine de plages.

11 Des doine tout à fait semblables ont été enregistrées en 1969 par l'auteur en Bucovine. L'excellente femme qui les a chantées se souvenait alors parfaitement du passage de Brǎiloiu dans son village et racontait qu'il lui avait été proposé à cette occasion d'aller à Bucarest pour essayer de se lancer comme professionnelle, ce à quoi elle s'était refusée.

Comme le précise Laurent Aubert, l'intérêt primordial du disque $C$ consacré à la Transylvanie est de fournir pour la première fois aux lecteurs assidus de l'œuvre complète de Brăiloiu - et surtout de sa célèbre monographie sur le répertoire du village de Drăgus ${ }^{2}$ - une illustration sonore abondante. Dix-neuf plages permettent, en effet, de se faire une idée assez précise de la constitution du répertoire de Drăgus. On y trouvera plusieurs sortes de chansons lyriques (qu'il est parfois difficiles de distinguer des doine), une très belle berceuse, toutes sortes de cris scandés pendant la cérémonie nuptiale ou pendant la danse (strigături), des chants de noce, des chants funèbres, un chant de moisson, un chant de quête des coutumes de Noël (colindă, genre qui a inspiré à Bartók sa Cantate profane), et diverses danses instrumentales pour clarinette seule ou pour ensemble à corde.

13 Dans l'ensemble comme dans le détail, ce coffret est une fort belle réalisation. Il s'adresse tant aux spécialistes qu'au grand public. Il est à recommander à tous ceux qui n'ont pu connaître de la musique traditionnelle roumaine que la face «folklorisée » que Marcel Cellier et Gheorghe Zamfir ont éclairée pendant des années avec trop de complaisance. Il 
complète - sans en oblitérer en aucune façon la valeur - les trois microsillons publiés dans la Collection CNRS/Musée de l'Homme par Le Chant du Monde - Harmonia Mundi (1985, Paris), et l'on ne peut que se réjouir de cet enrichissement.

Roumanie : Musique des Tsiganes de Valachie - Le taraf de Clejani. Un disque compact ADD publié sous le triple label Radio Télévision de la Suisse romande (RTSR), Archives internationales de musique populaire (AIMP, Genève) et OCORA Radio France. Distributeur Harmonia Mundi, Paris, février 1988. Enregistrement réalisé le 8 décembre 1986 dans le studio de l'Institut de recherches ethnologiques et dialectologiques (ICED) à Bucarest. Réalisation : Laurent Aubert, Thierry Fischer. Texte de présentation : Laurent Aubert. Conseiller scientifique : Speranza Rădulescu. Prise de son : Thierry Fischer.

Mis à part les repiquages dont il vient d'être question plus haut, et sauf erreur de ma part, c'est là le premier disque compact de musique roumaine paru à ce jour. C'est également la première fois qu'un disque longue durée est entièrement consacré au répertoire d'un taraf de village.

Il a fallu bien évidemment procéder à un choix, vu que les Tsiganes roumains ne peuvent être reconnus comme professionnels que s'ils sont capables de jouer plusieurs heures consécutives lors de noces qui peuvent durer jusqu'à trois jours. Ce choix a été judicieux. Quatre plages sont consacrées à de la musique vocale, le plus souvent préludée, interludée ou postludée par diverses pièces instrumentales de type «chant long» ou «mélodies de danse ", et une plage à de la musique de danse. Le tout forme un ensemble d'une belle cohérence.

Bien que les enregistrements aient été faits en studio, on se sent vraiment transporté dans une atmosphère de fête. Les musiciens sont des professionnels aussi à l'aise en studio que sur scène (comme on a pu s'en rendre compte lors de leurs récents passages à Paris et à Genève), et rien ne peut les empêcher de «chauffer ». Le plaisir d'écoute est donc fort grand.

La prise de son est d'excellente qualité, ce qui ne fait qu'accroitre l'extase. L'équilibre entre les diverses parties (solo/accompagnement poly-instrumental) est magnifique : on entend merveilleusement bien chaque instrument séparément, et l'ensemble sonne admirablement.

La notice très clairement rédigée fournit à l'auditeur des informations fondamentales sur la vie musicale du village de Clejani. Il est donc indispensable de la lire attentivement avant toute audition, si l'on veut se trouver dans les meilleures conditions d'écoute. Il est de la plus haute importance, en effet, pour apprécier ce disque à sa juste valeur, de savoir qu'il existe en Roumanie deux catégories de musiques de professionnels à laquelle celle des lăutari de Clejani n'appartient pas : la musique «folklorisée » ou «normalisée » (celle qui a été la plus «exportée » et qui est donc la plus connue), et la musique tsigane des faubourgs urbains (elle aussi assez bien connue). La musique des lăutari de village n'est donc pas celle à laquelle on accède le plus facilement lorsqu'on visite le pays en touriste. 


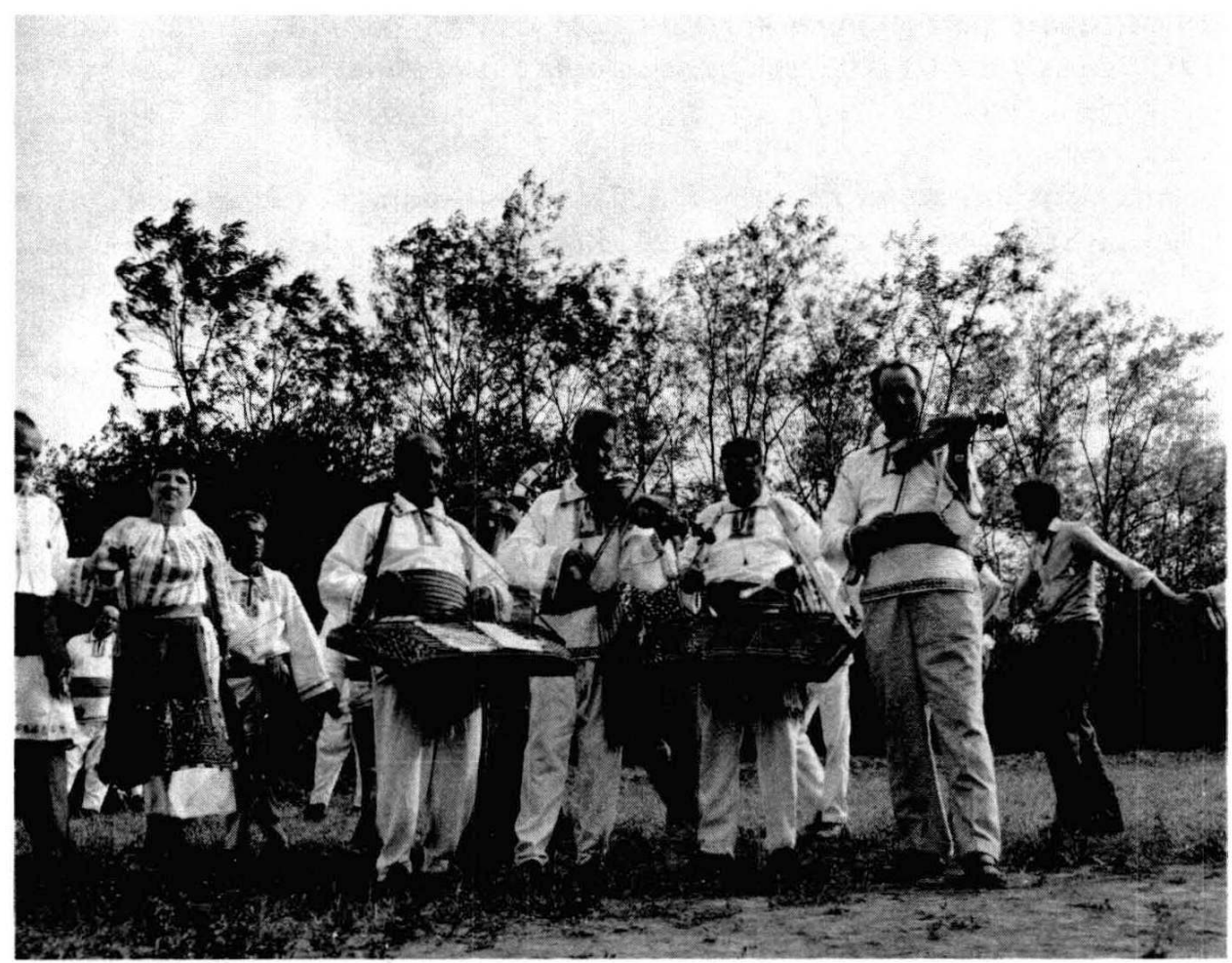

Photo : Pîrvu Emanuel, 1983.

Transylvanian Harmonies. Performers in the Past. Un microsillon (33 tours, $30 \mathrm{~cm}$ ) d'enregistrements effectués en 1941 par Constantin Brăiloiu et Tibe-riu Alexandru à Bucarest. Publication de l'Institut de recherches ethnologiques et dialectologiques (ICED) de Bucarest, sous la responsabilité éditoriale de Tiberiu Alexandru. Notice rédigée par Tiberiu Alexandru. The National Collection of Folklore, Disque ELECTRECORD, EPE 003319, Bucarest, 1984.

Ce très beau disque devrait éveiller l'intérêt de tous ceux qui sont sensibles aux sonorités de l'ensemble à cordes. Il plongera aussi dans l'amertume ceux qui savent que la musique transylvaine est menacée d'extinction depuis la décision récente prise par le chef de l'État roumain de détruire un village sur deux et de regrouper les habitants ainsi délogés dans des complexes agro-industriels urbanisés.

L'intention explicite de Tiberiu Alexandru a été de mettre en évidence un art de l'harmonisation propre aux lăutari des villages transylvains. Les ensembles à cordes transylvains sont, en effet, constitués d'un violon conducteur et d'une section d'accompagnement utilisant une contrebasse et un alto " préparé » appelé contra ou braci (de l'allemand Bratsche, «alto »). Cet alto (parfois remplacé par un violon ou deux) n'est pas monté ni accordé comme un alto classique. Il n'a que trois cordes ( $\mathrm{sol} / \mathrm{ré} / \mathrm{la})$, et son chevalet est rectiligne au lieu d'être courbe. Le musicien ne l'utilise que pour exécuter un accompagnement continu en accords de trois sons, le solo étant réservé exclusivement au violon. L'enchainement des accords ainsi joués en synchronie avec la contrebasse est soumis à des contraintes instrumentales qui donnent à l'harmonie une couleur très 
spécifique. C'est avant tout de la musique de danse qui est jouée dans le cadre de ces ensembles transylvains.

\section{NOTES}

1. Speranta Rădulescu, Taraful si acompaniamentul armonie în muzica de joc. Bucuresti : Edi-tura Muzicală, 1984.

2. Constantin Brăiloiu, Vie musicale d'un village. Recherches sur le répertoire de Drăgus (Roumanie), 1929-1932. Paris : Institut universitaire roumain Charles $1^{\mathrm{er}}, 1960$. 